\title{
Comparison of Clinical and Radiographic Periodontal Status between Hookah and Cigarette Smokers
}

\author{
Seyed Ali Banihashem $\operatorname{Rad}^{1}$, Mohammad Bagheri Iraj², Majid Sanat Khani ${ }^{3}$, Zahra Saeedi ${ }^{4}$, Seyed Ahmad \\ Banihashem Rad ${ }^{4}$, Hadi Yasamani ${ }^{4} \&$ Amir Movahhedian ${ }^{5}$ \\ ${ }^{1}$ Periodontology, Faculty of Dentistry, Mashhad University of Medical Sciences, Mashhad, Iran \\ ${ }^{2}$ Department of Prosthodontics, Faculty of Dentistry, North Khorasan University of Medical Sciences, Bojnurd, \\ Iran \\ ${ }^{3}$ Oral \& Maxillofacial Diseases Research Center, Mashhad University of Medical Sciences, Mashhad, Iran \\ ${ }^{4}$ Private Practice, Mashhad, Iran \\ ${ }^{5}$ Prosthodontics, Department of Prosthodontics, Tehran University of Medical Sciences, Tehran, Iran \\ Correspondence: Amir Movahhedian, Post graduate student of Prosthodontics, Department of prosthodontics, \\ Tehran University of Medical Sciences, Tehran, Iran. Tel: 4279-4000.
}

Received: March 30, 2020

doi:10.5539/jmbr.v10n1p176
Accepted: May 12, $2020 \quad$ Online Published: November 30, 2020

URL: https://doi.org/10.5539/jmbr.v10n1p176

\begin{abstract}
Background and Objective: Inhalation of chemicals and toxins in cigarette and hookah smoke results in loss of integrity of oral cavity tissues. The objective of this study was to compare the periodontal health of hookah and cigarette smokers.

Materials and Methods: In this study, 73 men at the age group of 20-35 years who smoked hookah for more than 5 years and 73 men at the same age who smoked cigarette for more than 5 years and 73 healthy men referred to the periodontal department of Mashhad dentistry school were selected and studied. Periodontal indices including pocket depth, GI, BOP, CAL were assessed in two groups. Also, using parallel periapical radiography, mesial and distal marginal bone level around the first molar tooth was measured. Finally, the data were assessed and compared using appropriate statistical analysis.

Results: Pocket depth was $24.27 \%$ and $23.62 \%$, respectively, in cigarette smokers and hookah smokers, and it was $0.96 \%$ in healthy subjects, clinical attachment level was 4.48 and $4.41 \mathrm{~mm}$, respectively, in cigarette smokers and hookah smokers, and it was $0.77 \mathrm{~mm}$ in healthy subjects. Gingival index (GI) was 1.40 and 1.42 , respectively, in cigarette smokers and hookah smokers and it was 0.52 in healthy subjects, BOP value was 6.52 and $6.52 \%$, respectively, in cigarette smokers and hookah smokers and it was $10.86 \%$ in healthy subjects. Mesial marginal bone level of first lower molar tooth was 2.27 and $2.32 \mathrm{~mm}$, respectively, in cigarette smokers and hookah smokers and it was $1.74 \mathrm{~mm}$ in healthy subjects and distal marginal bone level of lower first molar tooth was 2.38 and $2.35 \mathrm{~mm}$, respectively, in cigarette smokers and hookah smokers and it was $1.75 \mathrm{~mm}$ in healthy subjects. Comparison of results between the two groups of cigarette smokers and hookah smokers did not show any significant relationship in any of the variables, but there was a significant relationship between the two groups and the healthy group $(\mathrm{P}<0.001)$.
\end{abstract}

Conclusion: Periodontal parameters in healthy individuals are significantly better than those of smokers. Also, although there is a relationship between cigarette smoking and hookah smoking and periodontal parameters, these two groups do not show a significant difference in terms of periodontal parameters.

Keywords: Cigarette, Hookah, Periodontal Indices

\section{Introduction}

Periodontitis is an inflammatory disease of the supporting tissues of the tooth, caused by certain microorganisms, resulting in the progressive degradation of the periodontal ligament and the alveolar bone and formation of pocket, gingival degradation, or both. The clinical sign for the diagnosis of periodontitis is attachment loss level. This condition is usually associated with the formation of a pocket or gingival degradation and a change in the density and height of the adjacent alveolar bone (Nurman \& Takei, 2002). Periodontitis is in three forms: chronic, 
invasive, and as a manifestation of systemic diseases. Chronic periodontitis, as the most common of periodontitis, is slowly progressing and clinically important in adults, but may also be seen in adults. Chronic periodontitis is associated with plaque and usually progresses slowly to moderate, but more rapid degenerative periods may be seen (Nurman \& Takei, 2002; Kinane, Peterson, \& Stathopoulou, 2006). Specific clinical findings in patients with chronic periodontitis include the formation of upper gingival and sub-gingival plaque (which is often associated with germ formation), gingival inflammation, pocket formation, attachment loss, and alveolar bone resorption (Nurman \& Takei, 2002). It is a multifactorial disease and a major etiologic factor in the development of periodontal disease of microorganisms, but the disease progresses due to risk factors such as genetics, age, gender, local factors (improper dental restoration, structural form of tooth, root fractures, root canal segment resorption), systemic factors (HIV, diabetes), environmental factors (smoking, stress) and socioeconomic factors. Several studies have indicated that smoking is strongly associated with clinical criteria of periodontitis, including loss of tooth-holding bone and surrounding connective tissue (Kinane, Peterson, \& Stathopoulou, 2006; Tomar \& Asma, 2000). Cigarette smoking has been identified as a risk factor for the prevalence and progression of the periodontitis, which usually applies its effect through plaque bacteria and host response (Palmer \& Soory, 2003; Beck, Koch, Rozier, \& Tudor, 1990).

Smoking has nowadays become one of the health problems in different communities. It has been proven that smokers have poorer oral hygiene than non-smokers and are brushing their teeth less frequently (Tomar \& Asma, 2000; Kerdvongbundit \& Wikesjö, 2000). The increased incidence and severity of periodontal degradations associated with smoking indicates that routine host response to bacteria seen in chronic periodontal disease change in smokers and cause severe periodontal damages. This imbalance between the bacterium and the host response may be due to a change in the contents of the sub-gingival plaque or due to an increase in the virulence of the pathogenic organisms or a change in the rate of host responses, or all of them (Nurman \& Takei, 2002). More than 4000 toxics have been identified in cigarettes, including nicotine. Many of these substances are absorbed into the lung alveoli, but nicotine is absorbed by the oral mucosa. Nicotine is the first cause of the extensive effects of smoking on the human body, and its vascular effects largely explain the lack of ulcer healing (Mantilla Gomez, Danser, Sipos, Rowshani, Van der Velden, \& Van der Weijden, 2001). Tobacco smoking has a major effect on the parts protecting the immune system and its response, resulting in an increase in the extent and severity of periodontal damages. It seems that the harmful effects of tobacco use to be due to a weakening of the immune response in fighting against germs (Nurman \& Takei, 2002).

The pharmacological effects of nicotine vary, but the continuous vasoconstrictive ability of nicotine is a potential to reduce host defence and malnutrition in areas with low blood flow. The effect of nicotine on peripheral vessels is characterized by a reduction in the temperature of the fingertips and hands and feet of the smokers (Clarke \& Hirsch, 1995; Suter, Buzzi, \& Bättig, 1983). It has been reported that cigarette nicotine affect fibroblast and the production of collagen and non-collagen proteins is severely affected. Nicotine and carbon monoxide in cigarettes have a negative impact on ulcer healing. Cotinine in cigarettes also enhances the effects of toxins caused by periodontal pathogens, a mechanism that is involved in exacerbation of periodontal disease. Apatzidou et al. (2005) indicated that the inflammatory response in smokers decreased, although sub-gingival microbial flora in smokers was similar to that of non-smokers (Rivera - Hidalgo, 2003; Bergström, 2004; Apatzidou, Riggio, \& Kinane, 2005). The effect of smoking on the gingival tissue vessels can significantly decrease the symptoms of gingivitis in smokers compared to non-smokers, but the risk of development of periodontitis is twice as high as that of non-smokers in terms of age, gender, and economic status (Bergström \& Preber, 1986; Haber, Wattles, Crowley, Mandell, Joshipura, \& Kent, 1993). The risk of periodontitis in smokers is more than that of non-smokers. However, the risk of development of periodontitis decreases with the increase in the number of years that have passed since smoking (Nurman \& Takei, 2002; Preshaw, Heasman, Stacey, Steen, McCracken, \& Heasman, 2005). Histologic studies on smokers' ulcers showed fewer blood vessels in inflammatory ulcers compared to non-smokers (Rezavandi, Palmer, Odell, Scott, \& Wilson, 2002).

Polymorphonuclear (PMN) is the major defense cell in the gingival tissue and the first line of defense that phagocytizes invading bacteria in the dentogingival junction and contains enzymes such as elastase and collagenase that have been observed in the tissue structure of periodontitis. Tobacco use disrupts the function of neutrophils against periodontal infections or can increase the release of destructive tissue enzymes (Nurman \& Takei, 2002). Investigations have indicated a reduction in the migration of PMNs into the oral cavity and a reduction in phagocytosis capacity of PMNs in smokers compared to non-smokers (Palmer \& Soory, 2003). Smoking can weaken the defense of periodontal tissue by lowering serum vitamin C levels (Pelletier, 1975). Studies have indicated that smokers with periodontal disease have a higher number of sub-gingival of $\mathrm{T}$. forsythesis and P. gingivalis than non-smokers with the same level of periodontal disease. They also showed a 
slight reduction in the number of these bacteria after treatment than non-smokers. Various studies have shown that there is no difference between smokers and non-smokers in terms of rate of plaque formation, which may indicate that any change in smokers is related to qualitative changes in plaque not in quantitative ones (Nurman \& Takei, 2002; Grossi et al., 1997).

Smoking is also a predisposing factor for acute necrotizing ulcerative gingivitis (ANUG). In this disease, mucous membrane damage is a consequence of ascorbic acid deficiency and poor function of neutrophils. Environmental stressors reduce cellular immune function. Smoking reduces the effect of neutrophils and nicotine also causes vasoconstriction, leading to local tissue degradation (Goldhaber, 1964; Melnick, Roseman, Engel, \& Cogen, 1988). Pindburg in 1947 was one of the first researchers who investigated the association between smoking and periodontal disease. He reported the high prevalence of acute necrotizing ulcerative gingivitis (ANUG) in smokers. He also referred to an association between smoking and increased attachment loss and bone resorption (Pindborg, 1951). Due to an increasing number of smokers and given the cultural, social, economic and nutritional diversity among people, it is necessary to examine the effects of smoking on periodontal tissues. In recent years, the consumption of hookah, especially among the young population of the community has increased. Due to the lack of sufficient studies to compare these cases, the present study was conducted to compare the clinic and radiographic periodontal status of cigarettes and hookah smokers.

\section{Materials and Methods}

In the present study, 73 men aged 15-40 years who are smoking hookah for more than 5 years e and 73 at the same age who are smoking cigarettes for more than 5 years and 73 healthy controls referred to the periodontal department of Mashhad Dentistry School were studied. Before starting the study, informed consent was completed by all individuals. The smokers had to smoke at least 10 cigarettes daily, and the hookah smokers had to smoke hookah at least 3 times per week for at least 30 minutes each time. It should be noted that people who were smoking cigarettes and hookah simultaneously were excluded. Then, periodontal indices including pocket depth more than $3 \mathrm{~mm}, \mathrm{BOP}$ and CAL at six points (mesiobuccal, mid-buccal, distobuccal, distolingual/palatal, mid-lingual / palatal, and mesiolingual/palatal) on all available teeth, except for maxillary and mandibular third molars in three groups of subjects were studied and reported as percentage for BOP and pocket depth more than $3 \mathrm{~mm}$ and $\mathrm{mm}$ for CAL. Gingival index (GI) (Loe \& Silness) were also measured in all subjects and ranged from 0 to 3 (Figure 1). Also, using parallel periapical radiography, mesial and distal marginal bone level around the first mandibular molar was measured (the marginal bone was defined as the distance between the CEJ to the most coronal portion of the proximal bone in $\mathrm{mm}$ ). Finally, the obtained data were evaluated and compared using appropriate statistical analysis. This study was approved by the Ethics Committee of Mashhad Dentistry School.

\begin{tabular}{lll}
\hline $\begin{array}{l}\text { Scores } \\
0\end{array}$ & $\begin{array}{l}\text { Gingival Status } \\
\text { Normal gingiva }\end{array}$ & $\begin{array}{l}\text { Criteria } \\
\text { Natural coral pink gingival with no } \\
\text { e/o inflammation } \\
\text { Slight changes in color, slight } \\
\text { edema. No bleeding on probing }\end{array}$ \\
2 & Mild inflammation & $\begin{array}{l}\text { Redness, edema and glazing. } \\
\text { Bleeding upon probing }\end{array}$ \\
3 & Severe inflammation & $\begin{array}{l}\text { Marked redness and edema/ } \\
\text { ulceration/tendency to bleed } \\
\text { spontaneously }\end{array}$ \\
\hline
\end{tabular}

Figure 1. Gingival index (GI) (Loe \& Silness)

\section{Results}

In this study, 219 subjects were divided into 3 groups of hookah smokers, cigarette smokers, and control (each group included 73 subjects) and were investigated in terms of bleeding on probing (BOP), pocket depth (PD), clinical attachment level (CAL), height of mesial (HM), height of distal (HD) and gingival index (GI). The age range (oldest and youngest) was 20-35 years in the hookah and control groups and 21-35 years in the cigarette group.

The mean ages were $29.49 \pm 3.41,29.53 \pm 3.41$, and $29.27 \pm 3.38$ years, respectively, in hookah, cigarette, and control groups. The mean age was not statistically significant among the groups $(p=0.911)$. The normality of the data distribution was assessed by the Shapiro-Wilk test and it was found that data of quantitative variables in all 
groups followed a normal distribution $(\mathrm{P}<0.005)$. Based on Table 1 , all variables were significantly different among the three groups ( $\mathrm{P}<0.001$ for all variables). Pairwise comparison of the groups showed that in all variables, except for BOP, the means of the variables were not significantly different between the hookah and cigarette groups, but they were significantly lower in the control group compared to hookah and cigarette groups. The mean BOP variable was not significantly different between the hookah and cigarette groups, but it was significantly higher in the healthy group than that of the hookah and cigarette groups.

Table 1. Comparison of variables among the study groups

\begin{tabular}{lllllllll}
\hline Variable & Group & $\mathbf{N}$ & Mean & SD & Min & Max & Median & Kruskal-Wallis test result \\
\hline \multirow{4}{*}{ BOP } & Hookah & 73 & 6.52 & 4.95 & 0.0 & 20.0 & 5.0 & \multirow{2}{}{${ }^{2}=27.16$} \\
& Cigarette & 73 & 6.52 & 4.76 & 0.0 & 20.0 & 4.0 & $\mathrm{P}<0.001$ \\
PD & Healthy & 73 & 10.86 & 6.21 & 0.0 & 30.0 & 10.0 & \\
& Hookah & 73 & 23.62 & 7.20 & 10.0 & 42.0 & 24.0 & $\mathrm{X} 2=147.6$ \\
& Cigarette & 73 & 24.27 & 6.76 & 12.0 & 50.0 & 24.0 & $\mathrm{P}<0.001$ \\
CAL & Healthy & 73 & 0.96 & 1.53 & 0.0 & 5.2 & 0.0 & \\
& Hookah & 73 & 4.41 & 1.07 & 3.0 & 7.0 & 4.0 & $\mathrm{X} 2=144.6$ \\
& Cigarette & 73 & 4.48 & 0.84 & 3.0 & 7.0 & 4.0 & $\mathrm{P}<0.001$ \\
Hight.Mesial & Healthy & 73 & 0.77 & 1.17 & 0.0 & 4.0 & 0.0 & \\
& Hookah & 73 & 2.32 & 0.44 & 1.8 & 3.3 & 2.2 & $\mathrm{X} 2=113.6$ \\
Hight.Distal & Cigarette & 73 & 2.27 & 0.40 & 1.8 & 3.5 & 2.1 & $\mathrm{P}<0.001$ \\
& Healthy & 73 & 1.74 & 0.14 & 1.6 & 2.1 & 1.7 & \\
& Hookah & 73 & 2.35 & 0.39 & 1.8 & 3.4 & 2.3 & $\mathrm{X} 2=134.1$ \\
GI & Cigarette & 73 & 2.38 & 0.34 & 1.8 & 3.2 & 2.3 & $\mathrm{P}<0.001$ \\
& Healthy & 73 & 1.75 & 0.13 & 1.6 & 2.1 & 1.8 & \\
& Hookah & 73 & 1.42 & 0.88 & 0.0 & 3.0 & 1.0 & $\mathrm{X} 2=52.2$ \\
& Cigarette & 73 & 1.40 & 0.85 & 0.0 & 3.0 & 1.0 & $\mathrm{P}<0.001$ \\
\hline
\end{tabular}

In all three groups, age did not have a significant relationship with any of the variables. In the hookah group, BOP had a direct and significant relationship with PD, HM, HD and GI, but had a weak and non-significant and direct relationship with CAL. The PD variable had a direct and significant relationship only with the GI variable. Also, it had a direct but weak and non-significant relationship with CAL, HM and HD variables. The CAL variable had a direct and significant relationship with the HD and HM variables, but it had a direct, weak, and non-significant relationship with the GI. The HM variable had a direct and significant relationship with the HD and GI variables. The HD variable also had a direct and significant relationship with the GI.

In the cigarette group, BOP had a direct and significant relationship with PD, HM, HD and GI, but had a weak and non- significant relationship with CAL. The PD variable had a direct and significant relationship only with the HM and GI variables. Also, it had a direct but not significant relationship with CAL and HD variables. The CAL variable had a direct and significant relationship with the HD, HM and GI variables. The HM variable had a direct and significant relationship with the HD and GI variables. HD variable also had a direct and significant relationship with the GI variable. In the healthy group, BOP had a direct and significant relationship with PD and CAL, but a weak and non-significant and negative relationship with HD and HM variables, and a weak and non-significant and direct relationship with GI. The PD variable had a direct and significant relationship only with the variables of HM, CAL and GI. Also, it had a direct but weak and non-significant relationship with the HD variable. The CAL variable had a direct and significant relationship with the HD and GI variables, but it had a direct and weak and non-significant relationship with the HD variable. The HM variable had a direct and significant relationship with the HD variable, but it had a direct but weak and non-significant relationship with the HD variable. The HD variable also had a direct, weak, and non-significant relationship with the GI variable.

\section{Discussion}

Many studies have compared the periodontium of tobacco users with healthy subjects as a control group, but the present study is the first study conducted to investigate and compare these indices in cigarette smokers and hookah users. Results showed that the plaque index was lower in non-smokers compared to smokers. In this regard, contradictory results have been reported in previous studies. Based on the results of the studies conducted by Machuca et al. (Machuca, Rosales, Lacalle, Machuca, \& Bullón, 2000) and Al Wahadni and Linden (Al - 
Wahadni \& Linden, 2003), it was found that the mean plaque index was higher in non-smokers. Also, the results of a 10-year study conducted by Bergstrom et al. (Bergström, Eliasson, \& Dock, 2000) showed that the number of smokers decreased over time. In another study, the rate of plaque formation was similar in smokers and non-smokers. In general, the results of the present study indicated that the change that occurred in smokers compared to non-smokers was more related to qualitative changes in plaque. Zombon and Newman also reported that change in various microorganisms, such as Bacteroides forsythus, and especially in pockets less than $4 \mathrm{~mm}$, is so that more microorganisms can be observed in periodontal pockets of smokers. Therefore, the colonization rate of periodontal pathogens in smokers was more than that of non-smokers and this colonization can increase the prevalence of periodontal degradations (Nurman \& Takei, 2002; Zambon, Grossi, Machtei, Ho, Dunford, \& Genco, 1996). Also, levels of immunoglobulins, especially IgG2, against periodontal pathogens in smokers with periodontitis were lower than those of non-smokers, reducing their ability to cope with periodontal infections. In contrast, the level of gingival tumor necrosis factor-a, NFa, Prostaglandin E2 (PGE2) and elastagen in the smoker were higher, resulting in periodontal degradation. These reasons may justify the high correlation between cigarette smoking and periodontal disease (Boström, Linder, \& Bergström, 1998; Söder, 1999).

Based on the results of the present study regarding probing depth (PD), cigarette and hookah smokers had significantly more probing depths than healthy subjects. This result is in line with those of many studies, including Pindeburg (1947), which referred to an increase in the depth of pockets in smokers compared to non-smokers. In their study, Lafzi et al. conducted a clinical study on the effects of cigarette smoking on periodontal tissues of patients referred to Tabriz Dentistry School and reported that the probing depth was significantly higher in heavy smokers than that in non-smokers and mild smokers (Lafzi, Abolfazli, Eskandari, \& Shirmohammadi, 2007). Martinez et al. (1995) reported that PD increased significantly in heavy smokers compared to non-smokers. In a study conducted by Bergstrom et al. in 2000, PD showed a significant increase in smokers compared to non-smokers. In former smokers (those who used to smoke in the past), PD was also increased significantly compared to non-smokers. Among smokers, PD was higher in heavy smokers than that in light smokers. In a study conducted by Haffajee et al. in 2001, PD was significantly increased in smokers compared to non-smokers and former smokers (Haffajee \& Socransky, 2001). Also, in a study conducted by Clasina et al. (2002), the results showed a significant increase in PD in smokers compared to non-smokers (Calsina, Ramón, \& Echeverría, 2002).

In a study conducted to evaluate the effect of cigarette smoking on response to phase-one of periodontal treatment, Abolfazli et al. reported that there was a direct association between cigarette smoking and reduced clinical inflammation. Armitage et al. reported that with increasing the level of inflammation at the given site, with the same probing force, the probe penetrated more into the sulcus so that in periodontitis, the probe tip passed through the junctional epithelium and entered into the underlying connective tissue (Aboulfazli, Saleh Saber, Lafzi, \& Eskandari, 2007). The results of clinical attachment loss showed a significant increase in the level of attachment in cigarette and hookah smokers compared to healthy subjects. This result is in line with that of many studies, including Pindburg (Pindborg, 1951), in which the relationship between cigarette smoking and the level of attachment loss was reported. Also, based on the research carried out by Martinez et al. (1995), an increase in AL was observed in smokers. These results showed a significant increase in AL in heavy smokers compared to non-smokers. In comparing the non-smokers with light smokers, no significant difference was found between them. Also, based on the study conducted by Mullally et al. (1998), an increase in the level of attachment loss was observed in smokers compared to non-smokers. Based on the study conducted by Haffajee et al. (2001) and Klassina (Calsina, Ramón, \& Echeverría, 2002) in 2002, the mean level of attachment loss in smokers was higher than that of non-smokers and former smokers. The study conducted by Lafzi et al. in Tabriz also showed a significant increase in the level of attachment loss in heavy smokers compared to non-smokers and light smokers (Lafzi, Abolfazli, Eskandari, \& Shirmohammadi, 2007).

Nowadays, most smokers use toothpaste regularly to reduce the discoloration of their teeth. Higher abrasion of these toothpastes compared to the conventional toothpastes and the increased frequency of tooth brushing can decrease the plaque in smokers compared to non-smokers. Thus, it can be stated that if the smokers' personal health level is lower than that of non-smokers, they will have a higher plaque index. Also, we should refer to the effect of socioeconomic variables on smoking patterns and health status. Also, the plaque index of the patient is highly variable and depends on factors such as time, consumption or non-consumption of food and teeth brushing before the examination. A change in these factors might lead to different results in different studies (Liede, Haukka, Hietanen, Mattila, Rönkö, \& Sorsa, 1999; Pocock, Cook, Shaper, Phillips, \& Walker, 1987).

The results of investigating the bleeding on probing showed a significant difference between cigarette and hookah smokers compared to healthy people. The results of our study were in line with those of recent studies 
concocted in this regard. In a study conducted by Lafzi et al, a significant reduction in bleeding on probing was reported in heavy smokers compared to non-smokers and light smokers. In a study conducted by Bergstrom et al. in 2001, a significant reduction in probing on bleeding was observed in smokers compared to non-smokers and this result was more pronounced in patients with periodontitis compared to subjects with healthy gums (Bergström, 2004). Haffajee and Clasina also reported a reduction in this type of bleeding and gingivitis in smokers (Haffajee \& Socransky, 2001; Calsina, Ramón, \& Echeverría, 2002). Many studies have attributed this significant reduction in probing on bleeding in smokers to the decreased inflammatory response of the gum vessels and nicotine-induced vasoconstrictive property (Calsina, Ramón, \& Echeverría, 2002).

Studies have reported that nicotine has a vasoconstrictive effect on the blood vessels of gums, leading to suppression of bleeding on proving (BOP). In a clinical study conducted by Natto et al, subjects who smoked showed reduced gingival bleeding compared to control group subjects. The results of this study also are in line with those of clinical and laboratory studies conducted by Clarke, Shephard and Natto (Natto, Baljoon, \& Bergström, 2005; Clarke \& Shephard, 1984). In a study conducted by Abolfazli et al, probing on bleeding was $80 \%$ in non-smokers, $60 \%$ in light smokers, and $40 \%$ in heavy smokers at baseline before evaluating the effect of treatment on periodontal parameters. Heavy smokers had less bleeding on probation compared to non-smokers. It was due to changes in the structure of the small arteries of smokers compared to non-smokers, resulting in reduced blood flow and decreased inflammation-induced clinical symptoms (Aboulfazli, Saleh Saber, Lafzi, \& Eskandari, 2007). A study by Dietrich et al. (2004) also found that smoking reduced gingival bleeding. This effect was more in heavy smokers and lower in former smokers (Dietrich, Bernimoulin, \& Glynn, 2004).

Gingivitis has been reported lower in smokers compared to non-smokers in most of the studies (Kerdvongbundit \& Wikesjö, 2000; Bergström, Eliasson, \& Dock, 2000). Smoking seems to disrupt the chemotaxis, phagocytosis, and oxidative activity of neutrophils. Although no significant difference has been reported in the vascular density of healthy gums of smokers and non-smokers, it seems that that the microcirculation response to plaque accumulation to be different in smokers and non-smokers. These factors generally increase gingival fluid flow and bleeding on probing and decrease gingival blood vessels in smokers compared to non-smokers. However, it has been reported that this status to be inverse at a moderate level of inflammation (Bergström \& Preber, 1986; Persson \& Bergström, 1998). The results of a 10-year study conducted by Bergstrom et al. also confirm these results. Other studies have also reported that the rate of gingivitis is different in different states of mild to advanced gingivitis and periodontitis. Bergstrom and Bostrom also showed that bleeding on probing was lower in smokers than that in non-smokers. This study reported that the results were observed until the gum and disease conditions were the same in the subjects. Thus, with the same level of gum disease, smokers will have less gingivitis due to the mentioned reasons (Bergström \& Preber, 1986). The gingival index (GI) in the present study was 1.40 and 1.42 , respectively, in cigarette and hookah smokers. On average, all smokers had lower gingival health compared to non-smokers, confirming higher gingival index and bleeding on probing. Low gingival health in smokers is due to the fact that they pay less attention to oral health. However, when oral health is improved in smokers, gingival bleeding does not increase. However, studies have shown that the correlation between plaque and gingival bleeding in smokers is weaker than that in non-smokers.

The present study investigated marginal bone level and showed that cigarette and hookah smokers did not have any significant difference but showed slightly more reduction in bone level than the healthy group. The effect of smoking on marginal periodontitis has been well proven and it may be attributed to the indirect effects of poor oral health and poor general health status in smokers. It has been recently shown that smoking is a direct risk factor that reduces gingival bleeding, inhibits the immune response, and also changes the oral microflora towards pathologic. It has been indicated that smoking is directly correlated with degradation of periodontal tissues and marginal bone loss. Bahrami et al. in 2016 showed that smokers had a lower marginal bone level than non-smokers. The researcher reported that smoking had a statistically significant effect on the marginal bone level and smoking could be considered a real risk factor in marginal bone loss (Bahrami, Væth, Kirkevang, Wenzel, \& Isidor, 2017).

Pococl also showed that pockets above $5 \mathrm{~mm}$ and gingival attachment loss and periodontal disease were higher in smokers than non-smokers. Studies have shown that smoking increases bone resorption, increases pocket depth, increases the incidence of tooth decay, and increases the rate of gingival attachment loss (Pocock, Cook, Shaper, Phillips, \& Walker, 1987). This study revealed that pocket depth (PD), clinical attachment level, gingival index (GI), bleeding on probing (BOP), marginal bone level were almost similar in both groups. It has been well proven that hookah also exposes consumers to chemicals such as carbon monoxide, tar, and nicotine as much as cigarettes. Nicotine increases the secretion of pro-inflammatory cytokines such as interleukin-lbeta, which promotes alveolar bone loss. Also, smoking hookah once causes the maximum plasma concentration of nicotine, 
as much as smoking one cigarette. This may be a possible explanation for the similarity of periodontal parameters and marginal bone resorption in the two groups of hookah and cigarette smokers. Periodontal indices were not compared between cigarette and hookah smokers in the searched articles. The study conducted by Fawad Javed et al. was the only study compared to the two groups (Javed et al., 2016). Natto et al. evaluated the relationship between hookah smoking and periodontal indices. Their study revealed the effect of hookah smoking on the health of periodontal tissues was similar to that of cigarette smoking. Their study indicated that both forms of tobacco use (hookah and cigarette smoking) were associated with lower gum health. In both groups, there was a high incidence of periodontal pockets and decreased periodontal bone level compared to non-smokers (Natto, Baljoon, \& Bergström, 2005).

\section{Conclusion}

Periodontal parameters in healthy people are significantly better than that of smokers. Also, although there is a relationship between cigarette and hookah smoking and periodontal parameters, these two groups do not show a significant difference in terms of periodontal parameters.

\section{Conflict of interests}

The authors declare that there is no conflict of interests regarding the publication of this paper.

\section{References}

Aboulfazli, N., Saleh Saber, F., Lafzi, A., \& Eskandari, A. (2007). Effect of Cigarette Smoking Quantity on Periodontal Tissue Response to Phase I Therapy. Shiraz Univ Dent J., 8(3), 24-32.

Al-Wahadni, A., \& Linden, G. J. (2003). The effects of cigarette smoking on the periodontal condition of young Jordanian adults. Journal of Clinical Periodontology, 30(2), 132-7.

Apatzidou, D., Riggio, M., \& Kinane, D. (2005). Impact of smoking on the clinical, microbiological and immunological parameters of adult patients with periodontitis. Journal of Clinical Periodontology, 32(9), 973-83.

Bahrami, G., Væth, M., Kirkevang, L. L., Wenzel, A., \& Isidor, F. (2017). The impact of smoking on marginal bone loss in a 10-year prospective longitudinal study. Community Dentistry and Oral Epidemiology, 45(1), 59-65.

Beck, J. D., Koch, G. G., Rozier, R. G., \& Tudor, G. E. (1990). Prevalence and risk indicators for periodontal attachment loss in a population of older community-dwelling blacks and whites. Journal of Periodontology, 61(8), 521-8.

Bergström, J. (2004). Tobacco smoking and chronic destructive periodontal disease. Odontology, 92(1), 1-8.

Bergström, J., \& Preber, H. (1986). The influence of cigarette smoking on the development of experimental gingivitis. Journal of Periodontal Research, 21(6), 668-76.

Bergström, J., Eliasson, S., \& Dock, J. (2000). Exposure to tobacco smoking and periodontal health. Journal of Clinical Periodontology, 27(1), 61-8.

Boström, L., Linder, L. E., \& Bergström, J. (1998). Clinical expression of TNF- $\alpha$ in smoking-associated periodontal disease. Journal of Clinical Periodontology, 25(10), 767-73.

Calsina, G., Ramón, J. M., \& Echeverría, J. J. (2002). Effects of smoking on periodontal tissues. Journal of Clinical Periodontology, 29(8), 771-6.

Clarke, N. G., \& Hirsch, R. S. (1995). Personal risk factors for generalized periodontitis. Journal of Clinical Periodontology, 22(2), 136-45.

Clarke, N., \& Shephard, B. (1984). The effects of epinephrine and nicotine on gingival blood flow in the rabbit. Archives of Oral Biology, 29(10), 789-93.

Dietrich, T., Bernimoulin, J. P., \& Glynn, R. J. (2004). The effect of cigareté smoking on gingival bleeding. Journal of Periodontology, 75(1), 16-22.

Goldhaber, P. (1964). Present concepts concerning etiology and treatment of acute necrotizing ulcerative gingivitis. Int Dent J., 14, 468-96.

Grossi et al. (1997). Effects of smoking and smoking cessation on healing after mechanical periodontal therapy. The Journal of the American Dental Association, 128(5), 599-607. 
Haber, J., Wattles, J., Crowley, M., Mandell, R., Joshipura, K., \& Kent, R. L. (1993). Evidence for cigarette smoking as a major risk factor for periodontitis. Journal of Periodontology, 64(1), 16-23.

Haffajee, A., \& Socransky, S. (2001). Relationship of cigarette smoking to attachment level profiles. Journal of Clinical Periodontology, 28(4), 283-95.

Javed et al. (2016). Comparison of clinical and radiographic periodontal status between habitual water-pipe smokers and cigarette smokers. Journal of Periodontology, 87(2), 142-7.

Kerdvongbundit, V., \& Wikesjö, U. M. (2000). Effect of smoking on periodontal health in molar teeth. Journal of Periodontology, 71(3), 433-7.

Kinane, D. F., Peterson, M., \& Stathopoulou, P. G. (2006). Environmental and other modifying factors of the periodontal diseases. Periodontology 2000, 40(1), 107-19.

Lafzi, A., Abolfazli, N., Eskandari, A., \& Shirmohammadi, A. (2007). The Clinical Assessment of the Effects of Smoking on Periodontal Tissues in Referring Patients to Tabriz Dental Faculty during 2005-2006. Shiraz Univ. Dent. J., 7(3,4), 120-131

Liede, K. E., Haukka, J. K., Hietanen, J. H., Mattila, M. H., Rönkö, H., \& Sorsa, T. (1999). The association between smoking cessation and periodontal status and salivary proteinase levels. Journal of Periodontology 70(11), 1361-8.

Machuca, G., Rosales, I., Lacalle, J. R., Machuca, C., \& Bullón, P. (2000). Effect of cigarette smoking on periodontal status of healthy young adults. Journal of Periodontology, 71(1), 73-8.

Mantilla Gomez, S., Danser, M., Sipos, P., Rowshani, B., Van der Velden, U., \& Van der Weijden, G. (2001). Tongue coating and salivary bacterial counts in healthy/gingivitis subjects and periodontitis patients. Journal of Clinical Periodontology, 28(10), 970-8.

Martinez-Canut, P., Lorca, A., \& Magán, R. (1995). Smoking and periodontal disease severity. Journal of Clinical Periodontology, 22(10), 743-9.

Melnick, S. L., Roseman, J. M., Engel, D., \& Cogen, R. B. (1988). Epidemiology of acute necrotizing ulcerative gingivitis. Epidemiologic Reviews, 10, 191-211.

Mullally, B. H., \& Linden, G. J. (1996). Molar furcation involvement associated with cigarette smoking in periodontal referrals. Journal of Clinical Periodontology, 23(7), 658-61.

Natto, S., Baljoon, M., \& Bergström, J. (2005). Tobacco smoking and periodontal health in a Saudi Arabian population. Journal of Periodontology, 76(11), 1919-26.

Nurman, M., \& Takei, H. (2002). Carranza's clinical periodontology. Philadelphia: WB Saunders Company.

Palmer, R., \& Soory, M. (2003). Modifying factors: Diabetes, puberty, pregnancy and the menopause and tobacco smoking. Blackwell: Munksgaard.

Pelletier, O. (1975). Vitamin C and cigarette smokers. Annals NY Acad Sci., 258, 156-68.

Persson, L., \& Bergström, J. (1998). Smoking and vascular density of healthy marginal gingiva. European Journal of Oral Sciences, 106(5), 953-7.

Pindborg, J. J. (1951). Gingivitis in military personnel with special reference to ulceromembranous gingivitis. Odontol Tidskr, 59(6), 403-99.

Pocock, S., Cook, D., Shaper, A., Phillips, A. N., \& Walker, M. (1987). Social class differences in ischaemic heart disease in British men. The Lancet, 330(8552), 197-201.

Preshaw, P., Heasman, L., Stacey, F., Steen, N., McCracken, G., \& Heasman, P. (2005). The effect of quitting smoking on chronic periodontitis. Journal of Clinical Periodontology, 32(8), 869-79.

Rezavandi, K., Palmer, R., Odell, E., Scott, D., \& Wilson, R. (2002). Expression of ICAM-1 and E-selectin in gingival tissues of smokers and non-smokers with periodontitis. Journal of Oral Pathology \& Medicine, $31(1), 59-64$.

Rivera-Hidalgo, F. (2003). Smoking and periodontal disease. Periodontology 2000, 32(1), 50-8.

Söder, B. (1999). Neutrophil elastase activity, levels of prostaglandin E2, and matrix metalloproteinase-8 in refractory periodontitis sites in smokers and non-smokers. Acta Odontologica Scandinavica, 57(2), 77-82.

Suter, T. W., Buzzi, R., \& Bättig, K. (1983). Cardiovascular effects of smoking cigarettes with different nicotine deliveries. Psychopharmacology, 80(2), 106-12. 
Tomar, S. L., \& Asma, S. (2000). Smoking-attributable periodontitis in the United States: Findings from NHANES III. Journal of Periodontology, 71(5), 743-51.

Zambon, J., Grossi, S., Machtei, E., Ho, A., Dunford, R., \& Genco, R. (1996). Cigarette smoking increases the risk for subgingival infection with periodontal pathogens. Journal of Periodontology, 67, 1050-4.

\section{Copyrights}

Copyright for this article is retained by the author(s), with first publication rights granted to the journal.

This is an open-access article distributed under the terms and conditions of the Creative Commons Attribution license (http://creativecommons.org/licenses/by/4.0/). 